\title{
The Relationship between Stress and Mobile Phone Addiction in College Students
}

\author{
Minhui Zhang ${ }^{1^{*}}$, JunmingWei ${ }^{2}$ \\ ${ }^{l}$ Department of Applied Psychology, School of Public Administration, Guangdong University of Foreign Studies, \\ Canton, China \\ ${ }^{2}$ Shool of Physics, Shenzheng University, Canton, China
}

*Corresponding Author: Minhui Zhang, Department of Applied Psychology, School of Public Administration, Guangdong University of Foreign Studies, Canton, China

\begin{abstract}
In order to investigate the current situation of College Students' stress and mobile phone addiction and the relationship between them, college students were measured online with Perceived Stress Scale (PSS) and Mobile Phone Addiction Index (MPAI).The results showed that: (1) the degree of stress and mobile phone addiction of college students were at a medium level, the pressure of non-only child was higher than that of only child, and the pressure of junior students was higher than that of other grades. There were no differences in gender, age, grade, whether or not to be an only child, and major in mobile phone addiction among college students; (2) There was a significant positive correlation between stress and mobile phone addiction. The results of this study indicate that college students have a certain degree of stress and mobile phone addiction, and that stress is closely related to mobile phone addiction, which provides data support for understanding the mental health education of college students.
\end{abstract}

Key Words: mobile phone addiction, stress, college students, mental health education

\section{INTRODUCTION}

With the development of mobile Internet, smart phones are regarded as new or extended human organs by the media and the public. However, as modern people's "wind-accompanying ear" and "thousand-mile eye", mobile phones also cause social problems such as mobile phone addiction. Mobile phone addiction refers to the phenomenon of psychological or social function damage caused by excessive use of mobile phone. Excessive use of mobile phone will bring many physiological complications, such as neck, shoulder and other physiological pain, hearing and vision problems (Yen et al., 2009). In addition, mobile phone addiction has been proved to be related to people's dysfunctional behavior and negative personal status (Rozgonjuk et al., 2018), and it also has a negative impact on individual's academic performance and interpersonal relationship (Liu et al., 2017). In addition, mobile phone addiction has always been proved to be related to people's dysfunctional behaviors and negative personal status (Rozgonjuk et al., 2018), which will have a negative impact on individual academic performance and interpersonal relationships (Liu et al., 2017), and can cause mental health problems such as anxiety and depression (Huang et al., 2021).

Research on the influencing factors of mobile phone addiction has always been a hot topic in the field of psychology. Previous studies have found that the influencing factors of mobile phone addiction include demographic variables (age, gender, major, grade, regional differences, etc.), social interaction, personality characteristics, self-esteem $(\mathrm{Hu}, \& \mathrm{Li}, 2014)$, procrastination behavior (Rozgonjuk et al., 2018), loneliness(Liu, \&Wang, 2011), mental health level (Huang et al., 2013), sense of alienation (Jiang, \&Bai, 2014), self-control(Mei et al., 2017; Zhou, \& Zhou, 2017;Zhang et al., 2017;Zhang, Qin, \&Huang, 2019),etc. There are also studies found that stress has a significant impact on smartphone addiction (Cho et al., 2017; Deursen et al., 2015), which is one of the best predictors of mobile phone addiction behavior (Augner\& Hacker, 2012), and people with high stress are more likely to have mobile phone addiction behavior (Jeong et al., 2016). This result is consistent with the general strain theory (Jun \& Choi, 2015), that is, all kinds of tension or stress experienced by individuals will lead to negative experience and eventually lead to problem behaviors. Therefore, people who feel more 
pressure are more likely to be addicted to mobile phones (Liu et al., 2018).For college students, they have to face many pressures, such as adapting to the environment, completing their studies, interpersonal communication, making friends in love, entrepreneurship and employment, etc. these tasks are intertwined, which makes college students have a greater pressure load (Qinet al., 2020).According to a survey report on mobile phone use of Chinese college students released by Mycos Research Institute in 2018, more than $80 \%$ of college students are addicted to mobile phones. They use mobile phones for more than 5 hours a day, and $79 \%$ of them use mobile phones in class (Gao et al., 2020). Based on the above theory and empirical research, this study assumes that college students have a certain degree of stress and mobile phone addiction, and the stress is positively correlated with mobile phone addiction.

\section{METHOD}

\subsection{Participants}

Using the method of questionnaire survey, 411 valid questionnaires of Chinese college students with bachelor degree or above were collected through professional online questionnaire platform. As can be seen from table 1, women accounted for $77.6 \%$, non-only child accounted for $66.2 \%$, aged 16-25 accounted for $98.8 \%$, and senior students accounted for $36.3 \%$; The number of subjects majoring in literature and history is the largest, accounting for $44 \%$.

Table 1Subjects

\begin{tabular}{|l|l|l|l|}
\hline Item & Classification & Number & Percentage (\%) \\
\hline \multirow{4}{*}{ Gender } & Male & 92 & 22.4 \\
\cline { 2 - 4 } & Female & 319 & 77.6 \\
\hline \multirow{4}{*}{ Age } & Yes & 139 & 33.8 \\
\cline { 2 - 4 } & NO & 272 & 66.2 \\
\hline \multirow{5}{*}{ Grade } & $16-20$ & 171 & 41.6 \\
\cline { 2 - 4 } & $21-25$ & 235 & 57.2 \\
\cline { 2 - 4 } & $26-30$ & 3 & .7 \\
\cline { 2 - 4 } & Over 30 & 2 & .5 \\
\hline \multirow{5}{*}{ Major } & Freshman & 97 & 23.6 \\
\cline { 2 - 4 } & Sophomore & 67 & 16.3 \\
\cline { 2 - 4 } & Junior & 80 & 19.5 \\
\cline { 2 - 4 } & Senior & 149 & 36.3 \\
\cline { 2 - 4 } & Graduate and above & 18 & 4.4 \\
\hline & Science and Engineering & 83 & 20.2 \\
\cline { 2 - 4 } & Literature and history & 181 & 44.0 \\
\cline { 2 - 4 } & Arts & 12 & 32.9 \\
\cline { 2 - 4 } & other & 135 & \\
\hline
\end{tabular}

\subsection{Measures}

\subsubsection{Perceived Stress Scale}

The Perceived Stress Scale (PSS) developed by Zhu Linxian in 2007 was used. The scale has 21 questions, including five items: the stress of study, employment, interpersonal relationship, love and economic. The higher the score, the greater the stress. The Cronbach's $\alpha$ was 0.915 .

\subsubsection{Mobile Phone Addiction Index}

The Mobile Phone Addiction Index (MPAI) developed by Liang Yongzhi in 2008 was used as the mobile phone addiction index. There are 17 items in the scale. In order to use the scale more scientifically, after factor analysis, we deleted five questions in the scale, including the fifth to the seventh of out of control dimension, the fifth of abstinence dimension and the third of avoidance dimension, and finally established 12 items, which were divided into 4 factors: out of control (refers to the individual who spends a lot of time on the mobile phone and is difficult to control), abstinence (refers to the frustration when an individual cannot use the mobile phone normally), avoidance (refers to the use of mobile phone to avoid problems in real life) and inefficiency (refers to the decline of learning efficiency caused by improper use of mobile phone). The scale adopts Likert's five-point 
scoring method. The higher the score of the scale, the stronger the degree of mobile phone addiction. The Cronbach's $\alpha$ was 0.890 .

\subsection{Procedure}

The questionnaires were randomly distributed online through the questionnaire star platform. Informed consent was obtained from the participants before data collection. Participants were informed that their participation was voluntary and they could decline to participate at anytime they want.

\subsection{Data analysis}

SPSS20.0 software was used for data statistical analysis. The first step was to test the reliability and validity of the scale and make factor analysis to get more scientific conclusions; The second step was to describe and count the respondents' basic situation of using mobile phones, such as the time of using mobile phones, the occasions of using mobile phones, and the main uses of mobile phones; The third step was the descriptive statistics of the total and each dimension of stress and mobile phone addiction, namely mean and standard deviation; The fourth step was to use one-way ANOVA to test whether there were significant differences in demographic variables of the whole and each dimension of College Students' stress and mobile phone addiction; The fifth step was to test whether there was a correlation between stress and mobile phone addiction.

\section{Results}

\subsection{Reliability Analysis}

This study used SPSS 20.0 software's $\alpha$ reliability coefficient analysis method to analyze the reliability of the scale. The results showed that the $\alpha$ coefficient of stress was 0.915 and the $\alpha$ coefficient of mobile phone addiction was 0.890 , which indicates that the reliability of the scale is high and the internal reliability is consistent.

\subsection{Validity Analysis}

\subsubsection{KMO Value}

The validity of the questionnaire depends on the KMO value in the data analysis. The KMO value is between $0-1$, and the closer to 1 , the better the effect of factor analysis. The results showed that the KMO value of stress was 0.897 , and that of mobile phone addiction was 0.843 , which indicates that the scale has good validity and is suitable for factor analysis. The following will be exploratory factor analysis of stress and mobile phone addiction to test the validity of the questionnaire.

\subsubsection{Factor Analysis}

The rotation component matrix of stress showed that after rotation, the factor load values of most items were greater than 0.6 , and the factor load values of interpersonal pressure, employment pressure, love pressure and economic pressure were greater than 0.7 , which indicates that the convergence of stress data is very good. The rotation component matrix of mobile phone addiction showed that after rotation, the factor load value of each item was greater than 0.5 , and the factor load value of most items was greater than 0.7 , which indicates that the convergence of mobile phone addiction data is very good.

\subsection{The Current Situation of College Students' Mobile Phone Use and the Basic Situation of Various Variables}

\subsubsection{The current situation of college students' mobile phone use}

Table 2 showed that $64.5 \%$ of the respondents said that they used mobile phones for more than 4 hours a day, which indicates that modern college students use mobile phones for a long time; The vast majority of respondents often use their mobile phones before going to bed, going to the toilet and during rest and eating, so as to meet their needs of socializing, playing games, shopping, querying information and information.

Table2. Descriptive statistics of mobile phone use among College Students

\begin{tabular}{|l|l|l|l|}
\hline Item & Classification (Hour) & Number & Percentage $(\%)$ \\
\hline The time of using mobile & $0.5-1$ & 1 & 0.2 \\
\hline
\end{tabular}


The Relationship between Stress and Mobile Phone Addiction in College Students

\begin{tabular}{|l|l|l|l|}
\hline \multicolumn{4}{|l|}{} \\
phones & $1-2$ & 11 & 2.7 \\
\cline { 2 - 4 } & $2-3$ & 52 & 12.7 \\
\cline { 2 - 4 } & $3-4$ & 82 & 20.0 \\
\cline { 2 - 4 } & Over 4 & 265 & 64.5 \\
\hline \multirow{4}{*}{$\begin{array}{l}\text { The occasions of using } \\
\text { mobile phones }\end{array}$} & Before sleep & 375 & 91.2 \\
\cline { 2 - 4 } & Use the toilet & 240 & 58.4 \\
\cline { 2 - 4 } & Rest and eat & 258 & 62.8 \\
\cline { 2 - 4 } & Work & 81 & 19.7 \\
\cline { 2 - 4 } & Walk & 63 & 15.3 \\
\cline { 2 - 4 } phones & Other & 84 & 20.4 \\
\hline The main uses of mobile & Social contact & 381 & 92.7 \\
\cline { 2 - 4 } & Game & 177 & 43.1 \\
\cline { 2 - 4 } & Shopping & 226 & 55.0 \\
\cline { 2 - 3 } & Search for information, & 256 & 62.3 \\
\cline { 2 - 3 } & Read novels & 80 & 19.5 \\
\cline { 2 - 3 } & Other & 58 & 14.1 \\
\hline
\end{tabular}

\subsubsection{Basic Information of Each Variable}

The scales used in the study were all 5-point scales. It can be seen from table 3 that the subjects' stress was at the medium level $(M=2.36)$, mainly focusing on learning stress $(M=2.76)$ and employment stress $(\mathrm{M}=2.8)$; the subjects' mobile phone addiction was generally at a medium level $(\mathrm{M}=2.66)$, among which the scores for avoidance and inefficiency were the highest.

Table3. Descriptive statistics of each variable and its sub-dimensions $(N=411)$

\begin{tabular}{|c|c|c|}
\hline Variables & $M$ & $S D$ \\
\hline Stress(total) & 2.36 & 0.65 \\
\hline Study stress & 2.76 & 0.77 \\
\hline Interpersonal stress & 1.79 & 0.81 \\
\hline Employment stress & 2.80 & 1.02 \\
\hline Love stress & 1.80 & 0.96 \\
\hline economic stress & 1.86 & 0.96 \\
\hline Mobile Phone Addiction(total) & 2.66 & 0.81 \\
\hline Out of control & 2.34 & 0.86 \\
\hline Abstinence & 2.70 & 1.07 \\
\hline Avoidance & 2.93 & 1.19 \\
\hline inefficiency & 2.92 & 1.04 \\
\hline
\end{tabular}

\subsubsection{Analysis on the Differences of Stress and Mobile Phone Addiction among College Students}

In this study, independent sample t-test and one-way analysis of variance were used to test whether stress and mobile phone addiction were different in the demographics of gender, single child, age, grade, and major. The results showed that there was no significant demographic difference in mobile phone addiction. There were significant differences in stress on whether only child $(\mathrm{SIG}=0.006)$ and grade $(\mathrm{SIG}=.027)$ (see Table 4). Specifically, there were significant differences in economic stress variables on whether only child or not. The economic stress of non-only child was higher than that of only child; There were also significant differences in stress variables among different grades, among which the Junior respondents had the strongest sense of stress. (See Table 5 and table 6).

Table4. The difference test of each variable in demographic variables

\begin{tabular}{|c|c|c|}
\hline Demographic variables & $F$ & Stress \\
\hline Only child or not & Sig. (two-sided test) & 7.573 \\
\hline Grade & $F$ & .006 \\
\hline & Sig. (two-sided test) & 2.767 \\
\hline
\end{tabular}

Table5. The difference test of only child or not in stress variables

\begin{tabular}{|l|l|l|l|l|l|l|}
\hline & & Study stress & Interpersonal stress & $\begin{array}{l}\text { Employment } \\
\text { stress }\end{array}$ & Love stress & Economic stress \\
\hline Only child & $M$ & 2.65 & 1.71 & 2.60 & 1.82 & 1.58 \\
\hline & $S D$ & .76 & .77 & .96 & .95 & .80 \\
\hline Non-only child & $M$ & 2.82 & 1.83 & 2.90 & 1.79 & 2.01 \\
\hline
\end{tabular}


The Relationship between Stress and Mobile Phone Addiction in College Students

\begin{tabular}{|l|l|l|l|l|l|l|}
\hline \multicolumn{9}{|c|}{} \\
\hline & SD & .77 & .82 & 1.03 & .97 & 1.00 \\
\hline & Sig & .992 & .350 & .506 & .030 & .011 \\
\hline & $F$ & .000 & .874 & .444 & .862 & 6.582 \\
\hline
\end{tabular}

Table6. The difference test of grade in stress variables

\begin{tabular}{|l|c|c|c|c|c|c|c|}
\hline & $\begin{array}{c}\text { Freshman } \\
(M \pm S D)\end{array}$ & $\begin{array}{c}\text { Sophomore } \\
(M \pm S D)\end{array}$ & $\begin{array}{c}\text { Junior } \\
(M \pm S D)\end{array}$ & $\begin{array}{c}\text { Senior } \\
(M \pm S D)\end{array}$ & $\begin{array}{c}\text { Graduate and } \\
\text { above } \\
(\mathrm{M} \pm \mathrm{SD})\end{array}$ & $\begin{array}{c}\text { Sig. } \\
\text { (two-sided } \\
\text { test }\end{array}$ \\
\hline Stress & $2.28 \pm 0.68$ & $2.44 \pm 0.70$ & $2.47 \pm 0.63$ & $2.36 \pm 0.71$ & $1.98 \pm 0.39$ & 1.30 & 0.26 \\
\hline
\end{tabular}

\subsubsection{Correlation analysis of stress and mobile phone addiction in College Students}

Table 7 showed that there was a significant positive correlation between stress and mobile phone addiction. It suggested that the more stressed college students were, the more likely they were to become addicted to mobile phones.

Table7. Correlation coefficient scale of stress and mobile phone addiction $(N=411)$

\begin{tabular}{|c|c|c|}
\hline & 1 & 2 \\
\hline Stress & 1 & 1 \\
\hline Mobile phone addiction & $.564^{* *}$ & 1 \\
\hline
\end{tabular}

$* * p<0.01$.

\section{DISCUSSION}

\subsection{The Mobile Phone Addiction of College Students is in the Middle Level}

This study found that college students' mobile phone addiction was in the middle level, and there was no significant difference in gender, only child or not, age, grade and major. Previous studies have shown that there is a correlation between gender and the degree of mobile phone addiction. The degree of mobile phone addiction of female students is higher than that of male students (Cui et al., 2015). This is because female students will "stay" in dormitories more in their spare time, so they have a stronger sense of dependence on mobile phones. This is not consistent with the results of this study, which may be due to the large difference in the proportion of male and female subjects in this study, men only account for $22.4 \%$. In the future research, we should pay attention to the problem of gender proportion of subjects. In addition, there is no significant difference in population variables, which also proves that the phenomenon of mobile phone addiction of college students is very common, and the problem of mobile phone addiction is more serious.

\subsection{The Stress of College Students is in the Middle Level}

This study found that the stress of college students was in the medium level, and there were significant differences in grade and whether they were the only child or not. Among them, the stress level of the Juniorwas the highest, which may be related to the aggravation of learning tasks after entering the third year, and the severe choice of postgraduate entrance examination and employment, which was consistent with the previous research results (Yu,\& Deng, 2012). In addition, the economic stress of the non-only child was significantly higher than that of the only child, which was mainly because the only child can enjoy more material resources in the case of limited family resources, which has also been confirmed by relevant studies (Zhang,\&Liu, 2003).

\subsection{College Students' Stress and Mobile Phone Addiction Are Significantly Positively Correlated}

When at higher stress levels, individuals need to seek ways to relieve stress. As the carrier of various APPs, mobile phones can be used for reading, listening to music, watching film and television works, social networking, games, etc. It can be said that mobile phones are a high-efficiency, low-cost, and diversified stress relief tool, which also caters to meet the needs of college students who have no source of income. Some researchers believe that the main motivation behind addiction is to escape the stress and negative emotions in real life (Chuet al, 2020). As the pressure increases in the real world, the virtual world constructed by mobile phones may be beautiful, safe, and concealed, so college students may become addicted to it. This is in line with the results of previous studies, that is, when dealing with objective stress events, the greater the individual's psychologically perceived stress, the higher the risk of addiction to smartphones. On the contrary, the less the individual's psychologically perceived stress, the better Promote healthy mobile phone usage habits of college students (Qin et al., 
2020).

\section{CONCLUSION}

The results showed that: (1) the degree of stress and mobile phone addiction of college students were at a medium level, the pressure of non-only child was higher than that of only child, and the pressure of junior students was higher than that of other grades. There were no differences in gender, age, grade, whether or not to be an only child, and major in mobile phone addiction among college students; (2) There was a significant positive correlation between stress and mobile phone addiction.

This study found that college students had a certain degree of stress and mobile phone addiction, and that stress was closely related to mobile phone addiction. This provides data support for understanding the mental health education of college students. For example, in response to the high academic pressure and employment pressure of college students in this study, colleges and universities can provide psychologically flexible group counseling, career planning courses and employment guidance services. Of course, this study also has some limitations, including: First, horizontal research cannot draw clear causal inferences, so future research should use vertical design to further verify the relationship between stress and mobile phone addiction. Second, this study used Chinese college students as a sample, and women account for more than $70 \%$. The external validity of the research results needs to be verified. Future research needs to collect data through more channels to make the subjects more representative.

\section{ACKNOWLEDGEMENT}

This work was supported by The National Social Science Fund of China [grant number 16BSH094]

\section{REFERENCES}

[1] Augner, C., \& Hacker, G. W. (2012). Associations between problematic mobile phone use and psychological parameters in young adults. International Journal of Public Health, 57(2), 437-441. https://doi.org/10.1007/s00038-011-0234-Z.

[2] Cho, H. Y., Kim, D. J., \& Park, J. W. (2017). Stress and adult smartphone addiction: Mediation by selfcontrol, neuroticism, and extraversion. Stress and Health, 33(5), 624-630. https://doi.org/10.1002/smi.2749.

[3] Chu, X. Y., Huang, W. F., Li, Y., L. L.(2020). Relationship between Perceived Stress and Problematizing Excessive Online Gaming of Undergraduate: A Moderated Mediating Model. Chinese Journal of Clinical Psychology, 28(2), 379-382. https://doi.org/10.16128/j.cnki.1005-3611.2020.02.033

[4] Cui, Y. L.,Peng, M.,Han, Y. Y.,et al. (2015). Mobile Phone Dependence, Self-esteem and Loneliness in College Students. China Journal of Health Psychology, 23(8), 1193-1196. https://doi.org/10. 13342/j. cnki. cjhp. 2015. 08. 022

[5] Deursen, A. J. A. M. Van, Bolle, C. L., Hegner, S. M., \&Kommers, P. A. M. (2015). Modeling habitual and addictive smartphone behavior The role of smartphone usage types, emotional intelligence, social stress , self-regulation, age, and gender. Computers in Human Behavior, 45, 411-420. https://doi.org/10.1016/j.chb.2014.12.039.

[6] Gao, X. Q., Liu, C., Gao, G., \& Li, J. (2020). The effect of College Students' emptiness on mobile phone dependence: the mediating role of self-control. China Journal of Health Psychology, 28(10), 1548-1551. https://doi.org/10.13342/j.cnki.cjhp.2020.10.025.

[7] Hu, S.S., \& Li, L.Y. (2014). A review of influencing factors of mobile phone addiction. Science of Social Psychology, 29(5), 61-65.

[8] Huang, H., Zhou, C. Y., \& Yu, L. (2013). The relationship between mobile phone dependence and mental health of college students. Chinese Journal of School Health, 34(9), 1074-1076. https://doi.org/10.16835/j.cnki.1000-9817.2013.09.019.

[9] Jeong, S. H., Kim, H. J., Yum, J. Y., \& Hwang, Y. (2016). What type of content are smartphone users addicted to?: SNS vs. games. Computers in Human Behavior, 54, 10-17. https://doi.org/10.1016/j.chb. 2015.07.035.

[10] Jun, S., \& Choi, E. (2015). Academic stress and Internet addiction from general strain theory framework. Computers in Human Behavior, 49, 282-287. https://doi.org/10.1016/j.chb.2015.03.001.

[11] Jiang, Y. Z., \& Bai, X. L. (2014). The effect of mobile internet dependence on College Students' sense of Alienation: the role of social support system. Psychological development and education, 5, 540-549. https://doi.org/10.16187/j.cnki.issn1001-4918.2014.05.025.

[12] Leung, L. (2008). Linking psychological attributes to addiction and improper use of the mobile phone 
among adolescents in Hong Kong. Journal of Children and Media, 2(2), 93-113. https://doi.org/10.1080/17482790802078565.

[13] Liu, Q. Q., Zhang, D. J., Yang, X. J., Zhang, C. Y., Fan, C. Y., \& Zhou, Z. K. (2018). Perceived stress and mobile phone addiction in Chinese adolescents: A moderated mediation model. Computers in Human Behavior, 87, 247-253. https://doi.org/10.1016/j.chb.2018.06.006.

[14] Liu, H., \&Wang, H. L. (2011). Relationship between mobile phone addiction, loneliness and motivation of mobile phone use among college students. Psychological Science, 34(6), 1453-1457. https://doi.org/10.16719/j.cnki .1671 -6981.2011.06.017.

[15] Liu, Q. Q., Zhou, Z. K., Niu, G. F., \& Fan, C. Y. (2017). Mobile phone addiction and sleep quality in adolescents: Mediation and moderation analyses. Acta PsychologicaSinica, 49(12), 1524. https://doi.org/10.3724/sp.j.1041.2017.01524.

[16] Mei, S. L., Chai, J. X., Li, J. M., \& Wang, L. Y. (2017). Impulsivity, self-regulation and mobile phone dependence: the mediating role of mobile phone use. Studies of Psychology and Behavior, 15(1), 136-143.

[17] Qin, P. F., Zhao, S. Y., Li, D. L., Huang, M. M., \& Liu, G. Q. (2020). The effect of stress perception on mobile addiction: the sequence mediating effect of self-control and learning burnout. Journal of Psychological Science, 43(5), 1111-1116. https://doi.org/10.16719/j.cnki.1671-6981.20200512.

[18] Rozgonjuk, D., Kattago, M., \&Täht, K. (2018). Social media use in lectures mediates the relationship between procrastination and problematic smartphone use. Computers in Human Behavior, 89, 191-198. https://doi.org/10.1016/j.chb.2018.08.003.

[19] Yen, C. F., Tang, T. C., Yen, J. Y., Lin, H. C., Huang, C. F., Liu, S. C., \& Ko, C. H. (2009). Symptoms of problematic cellular phone use, functional impairment and its association with depression among adolescents in Southern Taiwan. Journal of Adolescence, 32(4), 863-873. https://doi.org/10.1016/j.adolescence.2008.10.006.

[20] Yu, Y., Deng, X. Z., Z. X. (2012). The Present Situation of College Students' Pressure Related Factors Research. JOURNAL OF WUHAN TEXTILE UNIVERSITY, 25(5), 46-48.

[21] Zhang,J. W., Liu, Y. X., J. S. (2003). A Practical Research into the Pressure among University Students and the Characteristic of the Solution. JOURNAL OF BEIJING INSTITUTE OF TECHNOLOGY, 5(1). https://doi.org/10.15918/j.jbitss1009-3370.2003.01.002

[22] Huang, Z. H., Tan, J. Y., Xu, H. F., Huang, B. Y., Chen, Q. S., Li, Z. X., Zhuang, W. M., Zhang, Y. B., \& Gao, Y. H.(2021). Association of smartphone addiction with daily behaviors and mental health during the COVID - 19 pandemic among medical college students. Chin J Sch Health, 42(5), 713-718. https:// doi.org/10.16835/j.cnki. 1000-9817. 2021.05. 018

[23] Zhou, E. Y., \& Zhou, H. Y.(2017). An empirical study on the relationship among subjective well-being, self-control and Internet addiction in college students. Journal of Graduate School of Chinese Academy of Social Sciences, 5, 17-24.

[24] Zhang, C., Zhai, L., \& Wang C. (2017). Mediating Effect of self-control the relationship between mobile phone dependence and academic procrastination in college students. China Journal of Health Psychology, 25(1), 145-148. https://doi.org/10.13342/j.cnki.cjhp.2017.01.034.

[25] Zhang, X. G., Qin, J., \& Huang, W. Y. (2019). The relationship between the sense of meaning of life and mobile phone addiction: the mediating role of self-control. Studies of Psychology and Behavior, 17(4), 536-545.

[26] Zhang, X., Wei, H., \& Ding, Q. (2019). Influence of stress on online game addiction of male college students: the mediating role of self-control. Studies of Psychology and Behavior, 17(5), 713-718.

[27] Zhu, L. X. (2007). Study on the mental stress and social support of undergraduate students and their relationships with learning burnout. [Master dissertation, Zhejiang University].

Citation: Minhui Zhang, JunmingWei. "The Relationship between Stress and Mobile Phone Addiction in College Students" International Journal of Humanities Social Sciences and Education (IJHSSE), vol 8, no. 7, 2021, pp. 286-292. doi: https://doi.org/10.20431/2349-0381.0807031.

Copyright: (C) 2021 Authors. This is an open-access article distributed under the terms of the Creative Commons Attribution License, which permits unrestricted use, distribution, and reproduction in any medium, provided the original author and source are credited. 